\title{
First demonstration of ARC-accelerated proton beams at the National Ignition Facility
}

Cite as: Phys. Plasmas 26, 043110 (2019); https://doi.org/10.1063/1.5085787

Submitted: 14 December 2018 . Accepted: 08 March 2019 . Published Online: 24 April 2019

D. Mariscal, T. Ma, S. C. Wilks, A. J. Kemp, C. J. Williams (D, P. Michel, H. Chen, P. K. Patel, B. A. Remington (D, M. Bowers, L. Pelz, M. R. Hermann, W. Hsing, D. Martinez (D), R. Sigurdsson, M. Prantil, A. Conder, J. Lawson, M. Hamamoto, P. Di Nicola, C. Widmayer, D. Homoelle, R. Lowe-Webb, S. Herriot, W. Williams, D. Alessi (D, D. Kalantar, R. Zacharias, C. Haefner, N. Thompson, T. Zobrist, D. Lord, N. Hash, A. Pak, N. Lemos (D), M. Tabak, C. McGuffey (D), J. Kim (D), F. N. Beg, M. S. Wei, P. Norreys, A. Morace, N. Iwata, Y. Sentoku (D), D. Neely, C. G. Scott, and K. Flippo (D)

ARTICLES YOU MAY BE INTERESTED IN

Computational modeling of proton acceleration with multi-picosecond and high energy, kilojoule, lasers

Physics of Plasmas 25, 083109 (2018); https://doi.org/10.1063/1.5040410

Progress toward a self-consistent set of 1D ignition capsule metrics in ICF Physics of Plasmas 25, 122704 (2018); https://doi.org/10.1063/1.5049595

Relativistic laser driven electron accelerator using micro-channel plasma targets Physics of Plasmas 26, 033110 (2019); https://doi.org/10.1063/1.5087409

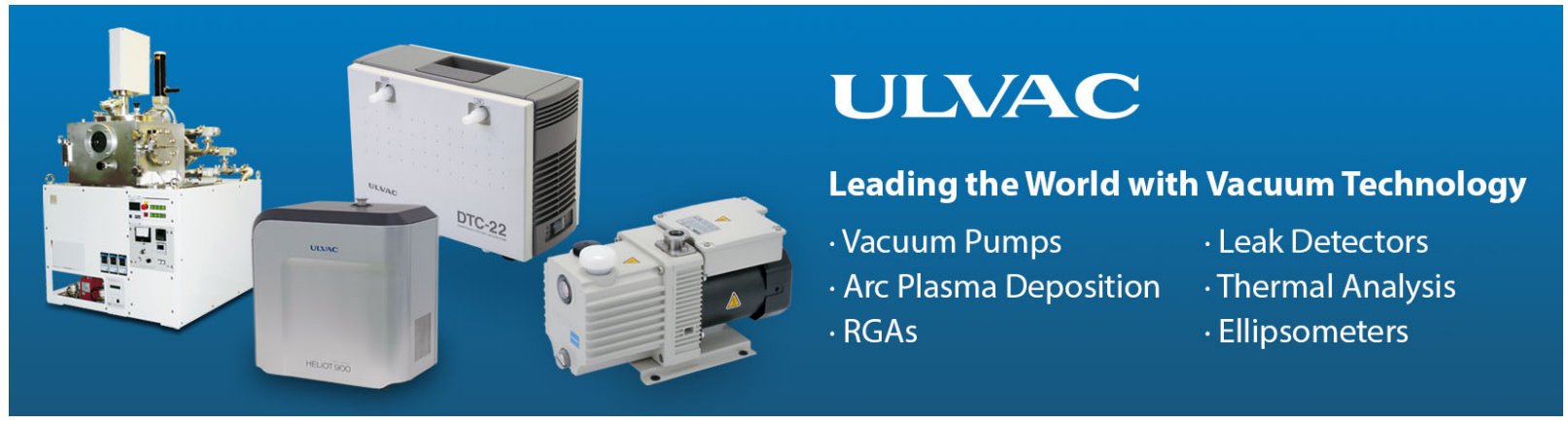




\title{
First demonstration of ARC-accelerated proton beams at the National Ignition Facility
}

\author{
Cite as: Phys. Plasmas 26, 043110 (2019); doi: 10.1063/1.5085787 \\ Submitted: 14 December 2018 - Accepted: 8 March 2019 . \\ Published Online: 24 April 2019
}

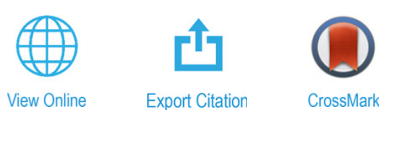

\author{
D. Mariscal, ${ }^{1, a)}$ T. Ma, ', S. C. Wilks, ' A. J. Kemp, ', G. J. Williams, \\ P. Michel, ${ }^{7}$ H. Chen, ${ }^{7}$ P. K. Patel, ${ }^{7}$ B. A. Remington, \\ M. Bowers, ${ }^{1}$ L. Pelz, ${ }^{7}$ M. R. Hermann, ${ }^{1}$ W. Hsing, ${ }^{7}$ D. Martinez, ${ }^{1}$ (D R. Sigurdsson, ${ }^{7}$ M. Prantil, ${ }^{7}$ A. Conder, J. Lawson, \\ M. Hamamoto, ${ }^{7}$ P. Di Nicola, ${ }^{\top}$ C. Widmayer, ${ }^{7}$ D. Homoelle, ${ }^{7}$ R. Lowe-Webb, ${ }^{7}$ S. Herriot, ${ }^{7}$ W. Williams, ${ }^{1}$ D. Alessi, ${ }^{1}$ \\ D. Kalantar, ${ }^{7}$ R. Zacharias, ${ }^{1}$ C. Haefner, ${ }^{7}$ N. Thompson, ${ }^{1}$ T. Zobrist, ${ }^{7}$ D. Lord, ${ }^{1}$ N. Hash, ${ }^{7}$ A. Pak, ${ }^{1}$ N. Lemos, ${ }^{1}$ iD M. Tabak, \\ C. McGuffey, ${ }^{2}$ (D) J. Kim, ${ }^{2}$ iD F. N. Beg, ${ }^{2}$ M. S. Wei, ${ }^{3}$ P. Norreys, ${ }^{4}$ A. Morace, ${ }^{5}$ N. Iwata, ${ }^{5}$ Y. Sentoku, ${ }^{5}$ (iD D. Neely, ${ }^{6}$ \\ G. G. Scott, ${ }^{6}$ and K. Flippo
}

\author{
AFFILIATIONS \\ 'Lawrence Livermore National Laboratory, Livermore, California 94550, USA \\ ${ }^{2}$ University of California at San Diego, La Jolla, California 92093, USA \\ ${ }^{3}$ General Atomics, La Jolla, California 92093, USA \\ ${ }^{4}$ University of Oxford, Oxford OX1 3PU, United Kingdom \\ ${ }^{5}$ Institute of Laser Engineering, Osaka University, Suita 565-0871, Osaka, Japan \\ ${ }^{6}$ Central Laser Facility, STFC Rutherford Appleton Laboratory, Didcot OX11 OQX, United Kingdom \\ ${ }^{7}$ Los Alamos National Laboratory, Los Alamos, New Mexico 87545, USA
}

Note: This paper is part of the Special Collection: Papers from the 60th Annual Meeting of the APS Division of Plasma Physics Note: Paper BI3 3, Bull. Am. Phys. Soc. 63 (2018).

a) Invited speaker.

\begin{abstract}
New short-pulse kilojoule, Petawatt-class lasers, which have recently come online and are coupled to large-scale, many-beam long-pulse facilities, undoubtedly serve as very exciting tools to capture transformational science opportunities in high energy density physics. These shortpulse lasers also happen to reside in a unique laser regime: very high-energy (kilojoule), relatively long (multi-picosecond) pulse-lengths, and large (10s of micron) focal spots, where their use in driving energetic particle beams is largely unexplored. Proton acceleration via Target Normal Sheath Acceleration (TNSA) using the Advanced Radiographic Capability (ARC) short-pulse laser at the National Ignition Facility in the Lawrence Livermore National Laboratory is demonstrated for the first time, and protons of up to $18 \mathrm{MeV}$ are measured using laser irradiation of $>1$ ps pulse-lengths and quasi-relativistic $\left(\sim 10^{18} \mathrm{~W} / \mathrm{cm}^{2}\right)$ intensities. This is indicative of a super-ponderomotive electron acceleration mechanism that sustains acceleration over long (multi-picosecond) time-scales and allows for proton energies to be achieved far beyond what the well-established scalings of proton acceleration via TNSA would predict at these modest intensities. Furthermore, the characteristics of the ARC laser (large $\sim 100 \mu$ m diameter focal spot, flat spatial profile, multi-picosecond, relatively low prepulse) provide acceleration conditions that allow for the investigation of $1 \mathrm{D}$-like particle acceleration. A high flux $\sim 50 \mathrm{~J}$ of laser-accelerated protons is experimentally demonstrated. A new capability in multi-picosecond particle-in-cell simulation is applied to model the data, corroborating the high proton energies and elucidating the physics of multi-picosecond particle acceleration.
\end{abstract}

Published under license by AIP Publishing. https://doi.org/10.1063/1.5085787

\section{INTRODUCTION}

The Advanced Radiographic Capability (ARC) laser of the National Ignition Facility (NIF) is a multi-picosecond, multi-kilojoule, large focal spot laser similar to the Omega EP laser at the University of Rochester, the LFEX laser at the University of Osaka, ${ }^{2}$ and the Laser MegaJoule's PETAL system. ${ }^{3}$ ARC intensities are nominally subrelativistic due to large focal spots, but the total deliverable energy is large and the pulse-length is long, resulting in a testbed for studying 1D-like laser plasma interactions. Current single beamlet average intensities are around $8 \times 10^{17}$ and $2 \times 10^{18} \mathrm{~W} / \mathrm{cm}^{2}$ for the 10 and 1 ps pulse-lengths, respectively. The reason for the low intensities is primarily the limited ability to focus on which produces a large focal spot containing 50\% of the laser energy in an ellipse 
with minor and major distances of approximately $30 \times 70 \mu \mathrm{m}$. An advantageous feature of the ARC laser is that it is very well characterized due to the extensive diagnostics at the ARC Diagnostic Table (ADT). The ADT is located downstream from the compressor gratings and measures the near and far field, wavefront, prepulse contrast, energy, spectrum, and pulse timing for one of the four beamlets during any given shot, and these data are used to validate models which predict the pulse shape and the time varying focal spot. An example of some of these data is shown in Fig. 1(c), where the focal spot has been modeled. These measurements and modeling enable detailed characterization of laser delivery for all four beamlets. For more details on the ARC laser characteristics and diagnostics, the reader is referred to Ref. 4 .

Coupled with the NIF, developing ARC laser driven ion acceleration capabilities will enable multiple exciting applications. For example, the NIF can deliver $1.8 \mathrm{MJ}$ of laser light to drive an experiment and with an energetic proton beam, we could begin to diagnose electromagnetic fields in these experiments by using proton radiography. ${ }^{5}$ With four independently pointed and timed beamlets available, this could enable diagnosing plasmas from four independent lines of sight at four separate times. Another application envisioned would be proton isochoric heating, where a proton beam containing 10s of Joules of energy promptly deposits its energy in a sample, heating it to 100s of electron volt temperatures before it has time to hydrodynamically respond. ${ }^{6}$ Intense proton beams can also be used to investigate the stopping of light ions in materials. ${ }^{7}$ By accelerating deuteron beams, ${ }^{8}$ nuclear reaction experiments could be conducted which could potentially drive on-demand neutron beams that are produced on a picosecond time-scale. Importantly, NIF's diagnostic suite enables extremely detailed measurements of these types of experiments.

While there are a number of ion acceleration schemes, perhaps the most familiar is the so-called target normal sheath acceleration mechanism. ${ }^{10}$ It has long been known that 10 s of $\mathrm{MeV}$ protons can be accelerated from intense $\left(>10^{19} \mathrm{~W} / \mathrm{cm}^{2}\right)$, sub-picosecond laser interactions with solid targets. This is a relatively simple mechanism that can be described in three stages. When a short pulse laser is incident on a thin micron-scale foil, mega-electron volt energy electrons can be accelerated, which are energetic enough to pass through the thin solid-density target. An electric field (or sheath field) is then generated on the rear surface due to charge separation. This electric field is then proportional to the hot electron temperature divided by the local density scale-length, $E[\mathrm{MeV} / \mu \mathrm{m}]$ $\propto T_{h o t} / e L_{n}$. Finally, hydrocarbon contaminants, which are present on the surfaces of the foil, are rapidly accelerated to multi-megaelectron volt energies. Protons possessing the highest charge-tomass ratio are preferentially accelerated by this field. Thus, $\mathrm{MeV}$ electron temperatures and energies are needed to generate multimega-electron volt proton energies.

There are many mechanisms that can result in these high energy electrons; however, in intense, sub-picosecond laser plasma interactions, the ponderomotive force is a dominant source of electron acceleration. ${ }^{11}$ Here, the laser pulse interacts with plasma densities near the critical density surface in a micron-scale-length preplasma and a gradient in the laser intensity, or the electric field, which is always present due to the finite rise in the laser intensity $I_{L}$, gives rise to the so-called ponderomotive force, $F_{p}=-\frac{e^{2}}{4 \pi \omega^{2}} \nabla E_{o}^{2}$, which gives electrons an energetic push in the forward direction. Here, $e$ is the electron charge, $\omega$ is the light frequency, and $E_{o}$ is the laser electric field amplitude. The hot electron temperature then scales with the laser intensity, $l_{L}$, as

$$
T_{\text {hot }}[\mathrm{MeV}]=0.511\left(\sqrt{1+I_{L} \lambda_{\mu m}^{2} / 2.74 \times 10^{18}}-1\right) .
$$
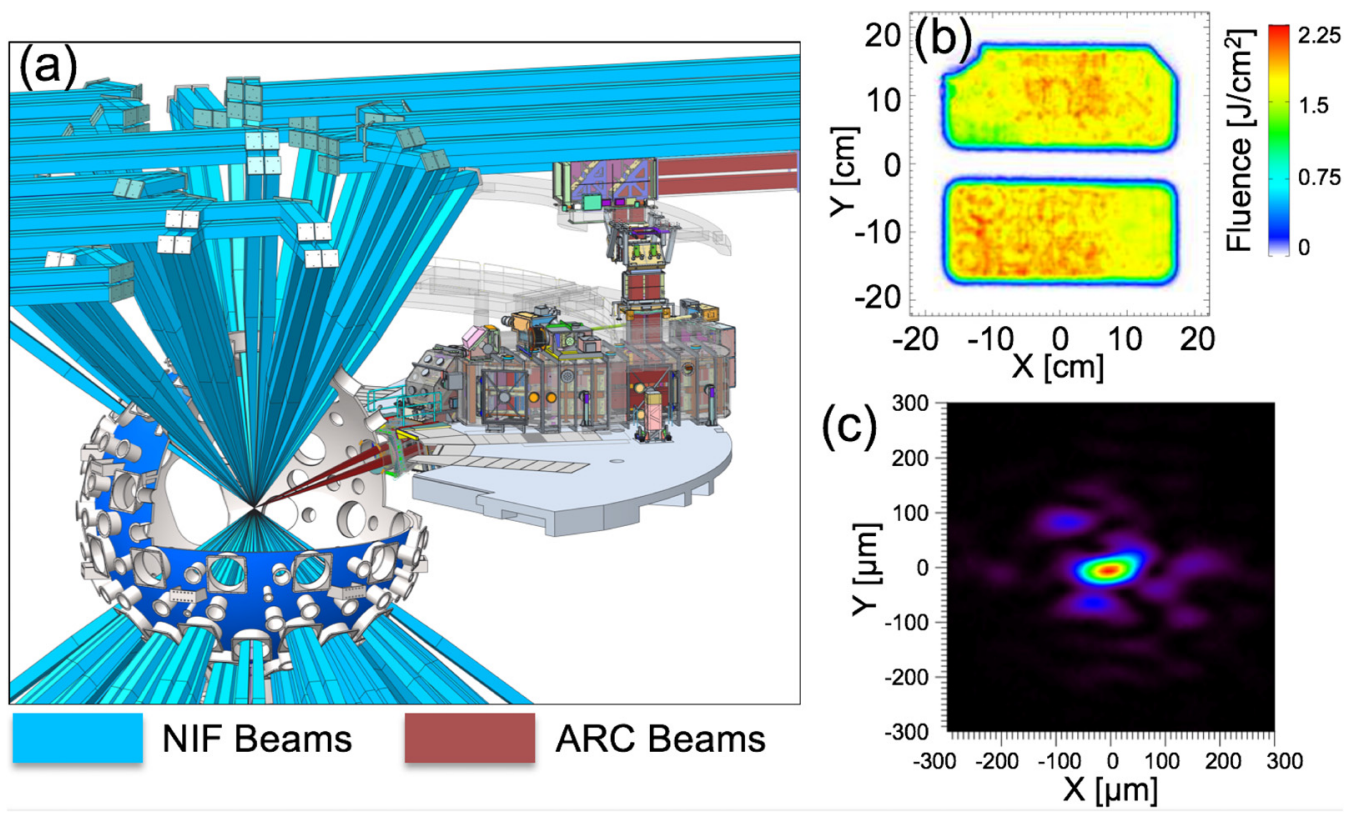

FIG. 1. (a) A schematic of the NIF target chamber, 192 NIF long-pulse beams shown in blue, and two of the NIF long pulse beams picked off for ARC shown in red. (b) The two long pulse beams are apertured to form two rectangular beamlets each, giving a total of four beamlets which are compressed to picosecond pulse-lengths. (c) The modeled ellipsoidal focal spot for one of the four beamlets at TCC. 
This scaling has been verified over a large number of short pulse experiments and facilities. For short pulse-lengths, small preplasma scale-lengths, and near-critical densities, the electron temperature is well described by this scaling. In interactions with large focal spots, like ARC, significantly longer scale-length preplasmas can be sustained. A simple picture shown in Fig. 2 illustrates that the radius of the laser focal spot essentially sets the maximum preplasma scalelength since plasma cooling will occur once the expansion toward the laser is approximately equal to the radius of the focal spot, at which point the assumption of 1D-like plasma expansion no longer applies. For a typical short-pulse laser experiment, the focal spot is on the order of $5 \mu \mathrm{m}$ radius, where in the case of ARC, the radius is $30 \mu \mathrm{m}$ or greater, meaning that $1 \mathrm{D}$-like expansion can be sustained for greater than $10 \mu \mathrm{m}$ such that there is a significant amount of underdense plasma for the laser to interact with before it reaches the critical density surface. When a long scale-length preplasma exists, electrons can be accelerated to energies which significantly exceed those predicted by the ponderomotive scaling via multiple mechanisms. For example, self-modulated laser wakefield acceleration can occur, where the laser can modulate plasma waves that allow electrons to be accelerated to super-ponderomotive energies. ${ }^{12}$ Similarly, direct laser acceleration (DLA) can occur when plasma channels are formed in this underdense plasma. ${ }^{13,14}$ The development of a potential well due to a plasma gradient in the low-density preplasma can result in electron dephasing and acceleration to high energies. ${ }^{15,16}$ There are several other mechanisms which can also result in "super-ponderomotive" electrons, and all these mechanisms can accelerate electrons to energies that are in many cases significantly $(>10 \times)$ greater than those accelerated by the ponderomotive force. As discussed earlier, proton acceleration to $>10 \mathrm{MeV}$ energies via TNSA relies heavily upon the generation of multi-mega-electron volt electron energies.

\section{FACILITY AND EXPERIMENTAL SETUP}

Two shots were conducted at 10 and 1 ps pulse-lengths in order to investigate a pulse-length and energy scan. The targets were $1.5 \times 1.5 \mathrm{~mm}$ square, $33 \mu \mathrm{m}$ thick Titanium foils. All four of the ARC beamlets were incident on the target and spatially separated by around $500 \mu \mathrm{m}$, as seen in Fig. 3(a), in order to minimize uncertainties in delivered laser intensity due to potential pointing and/or timing errors. As reported by the ADT, approximately $650 \mathrm{~J}$ per beamlet was delivered in a 9.6 ps pulse giving a total of $2.6 \mathrm{~kJ}$ on the target, while $275 \mathrm{~J}$ per beamlet was delivered at a $1.6 \mathrm{ps}$ pulse-length, giving a total of $1.1 \mathrm{~kJ}$ on the target. The average intensities were $9.1 \times 10^{17} \pm 1.9 \mathrm{~W} \mathrm{~cm}^{-2}$ and

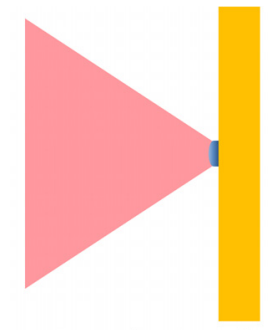

$\mathrm{r}_{\mathrm{L}}<5 \mu \mathrm{m} \rightarrow<5 \mu \mathrm{m} \mathrm{L}_{\mathrm{pp}}$

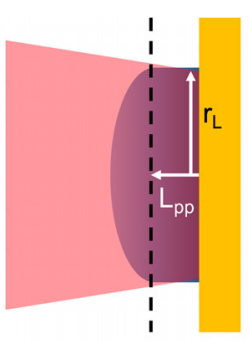

$r_{L}>30 \mu m \rightarrow>10 \mu m L_{p p}$
FIG. 2. A schematic showing a comparison of preplasma scale-length development vs laser focal spot size for small focal spots typical of short-pulse high-intensity experiments (left) vs large focal spots like ARC (right).

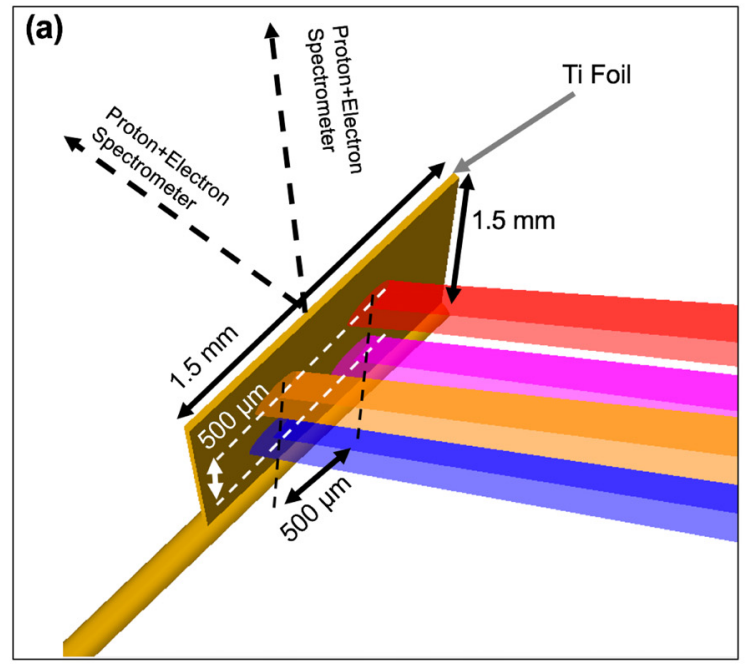

(b)

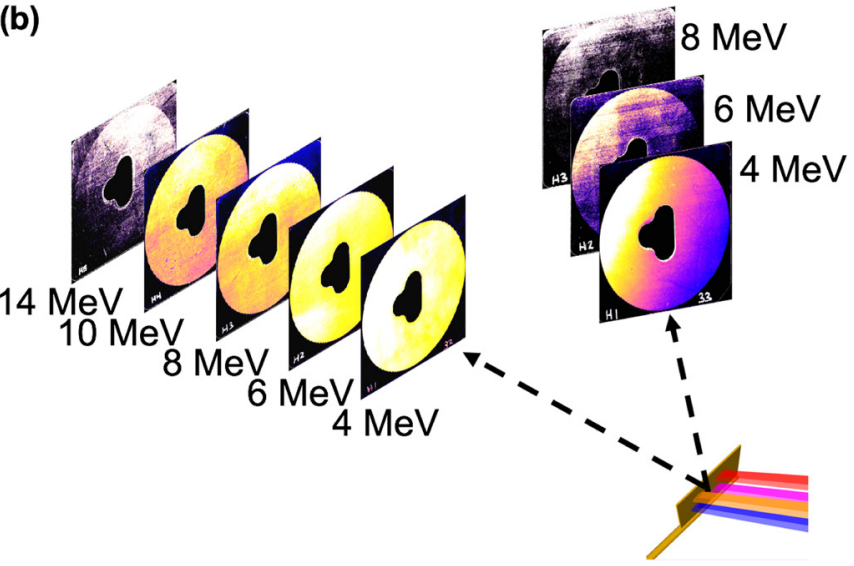

FIG. 3. (a) A zoomed in view showing the target foil, ARC beamlet pointing, and direction to the primary diagnostics. (b) Example of RCF stack data placed at the front of the NEPPS instruments.

$1.5 \times 10^{18} \pm 0.4 \mathrm{~W} \mathrm{~cm}^{-2}$ for the 10 and 1 ps pulse-lengths, respectively, where the uncertainty quoted refers to the population statistics for all four beamlets. Peak intensities were roughly twice the average value with approximately $30 \mathrm{~J}$ per beamlet of laser energy at or above this intensity for either pulse-length.

All available electron and proton diagnostics were fielded on each shot including a NIF Electron Positron Proton Spectrometer (NEPPS) magnetic spectrometer, ${ }^{17,18}$ a Magnetic Recoil Spectrometer (MRS), ${ }^{19}$ and multiple Wedge Range Filters (WRFs) ${ }^{20}$ in order to capture absolute electron and proton spectra and directionality. Multiple X-ray diagnostics were also fielded in order to diagnose the spatial and temporal laser delivery to the target. The NEPPS diagnostics provided the primary measurements, with one along the target normal direction and the other $33^{\circ}$ from this axis. While these spectrometers use a static $\mathrm{B}$-field to disperse particles for recovering continuous particle spectra, it is possible, additionally, to run radiochromic films (RCFs) at the front of the diagnostic. Radiochromic film stacks are often used in these types of experiments in order to get both spectral and spatial 
information about the proton beams. ${ }^{21}$ Some examples of RCF data are shown in Fig. 3(b) for the 10 ps pulse-length. The film stack is far from the source, such that the radiochromic film is over-filled and a circular aperture of the film holder is apparent along with a large hole in the center of the films which allows particles to freely enter the particle spectrometer. The last film, which observed proton signals along the target-normal direction, is used to define the maximum proton energy for these experiments, where maximum proton energies of 14 and $18 \mathrm{MeV}$ were observed for the 10 and 1 ps pulse-lengths, respectively. The continuous spectral measurements, seen in Fig. 4(b), were used to infer an extremely large flux of $\sim 50 \mathrm{~J}$ at energies greater than $3.5 \mathrm{MeV}$ at the $10 \mathrm{ps}$ pulse-length. Due to the inability to sample the full proton beam, spatial profile measurements from each film stack (placed at $0^{\circ}$ and $33^{\circ}$ from the target normal) and the MRS diagnostic ( $29^{\circ}$ from the target normal) were used to reconstruct the beam divergence scaling and thus the total energy contained in the proton beam. This amount of energy contained in a proton beam should enable access to extremely exotic states of matter at high temperature and near solid density if employed for a proton isochoric heating platform. The measured slope of the escaping electrons is actually about five to ten times higher than that expected from ponderomotive, whereas the effective slope temperature is approximately $1 \mathrm{MeV}$ for both pulselengths. This can be seen in Fig. 4(a) where the experimental data are

(a)

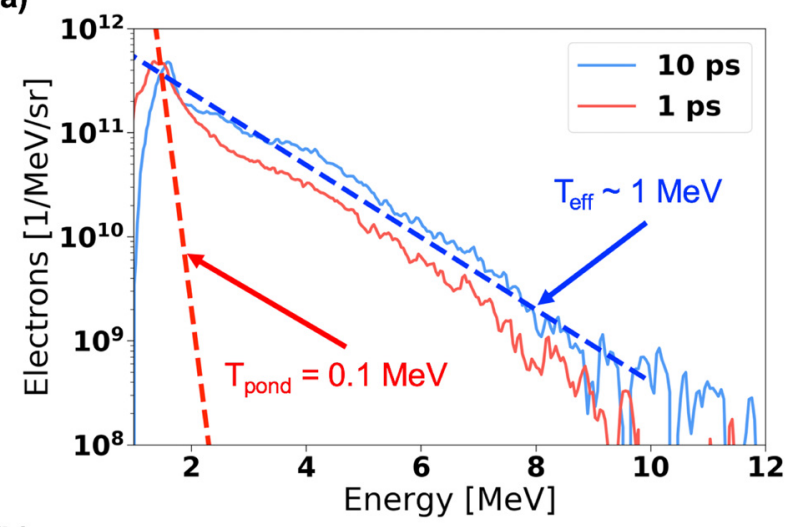

(b)

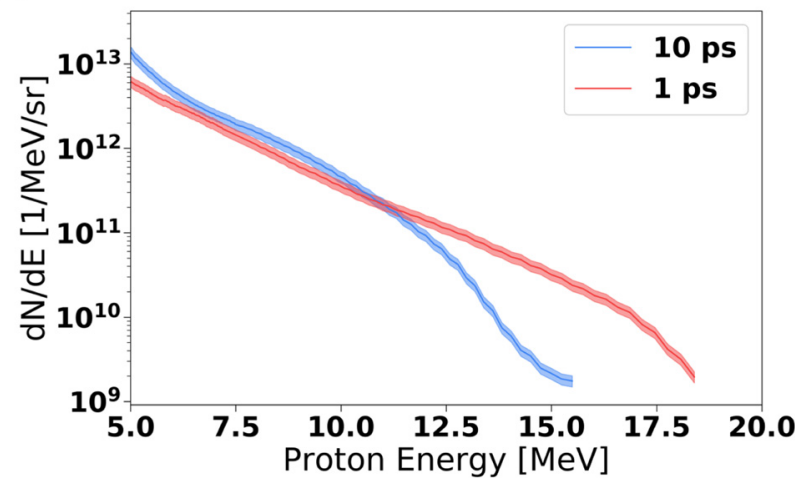

FIG. 4. (a) Electron and (b) proton spectra measured using the NEPPS diagnostic along the target-normal direction for the 1 and 10 ps pulses. plotted along with a line near the expected ponderomotive temperature for these laser intensities $(\sim 0.1-0.2 \mathrm{MeV})$ and a line with a $1 \mathrm{MeV}$ slope which is the effective hot electron temperature.

\section{TNSA SCALING}

A typical maximum proton energy scaling was presented by Fuchs, ${ }^{22}$ which is essentially a modification of an isothermal plasma expansion model. ${ }^{23}$ The scaling for the maximum proton energy is $E_{\text {max }}=2 T_{\text {hot }}\left[\ln \left(t_{p}+\left(t_{p}^{2}+1\right)^{1 / 2}\right)\right]^{2}$, where $t_{p}=\omega_{p i} t_{a c c} / \sqrt{2 e}$ is the normalized acceleration time (where $\left.\omega_{p i}=\left[\left(Z_{i} e^{2} n_{e 0}\right) /\left(m_{i} \epsilon_{0}\right)\right]^{1 / 2}\right), Z_{i}$ is the charge number, $m_{i}$ is the ion mass, $e$ is the electron charge, $\epsilon_{0}$ is the electric permittivity, and $n_{e 0}$ is the density of the hot electrons that drive the rear surface expansion. A key parameter is the effective acceleration time, $t_{p}$, which is dependent upon a free parameter, $t_{a c c}$, which is set to $\sim 1.3 \tau_{\text {laser }}$ for good agreement with experimental data. The parameter $\tau_{\text {laser }}$ gives a clear dependence and benefit to longer pulselengths for a given intensity. Another key feature of this scaling is that the hot electron temperature is taken to be the ponderomotive temperature scaling from Eq. (1). Here, it is clear that $>1 \mathrm{MeV}$ electron temperatures are reached when the intensity exceeds several $10^{19} \mathrm{~W} / \mathrm{cm}^{2}$ for $1 \mu \mathrm{m}$ laser light, and thus, most experiments focus on increasing the laser intensity to achieve higher proton energies. The green band in Fig. 5 is the Fuchs scaling plotted for a wide range of intensities for pulse-lengths ranging from 50 to $700 \mathrm{fs}$ along with experimental data in this range. From this, it is clear that this scaling works well for typical sub-picosecond pulse-length experiments. The blue and red lines show the predictions using this scaling for 10 and 1 ps pulses, respectively, and the maximum proton energy for either ARC pulse-lengths is predicted to be $\sim 4 \mathrm{MeV}$. Of course, the observed proton energies for ARC experiments are seen to significantly exceed this scaling by 3 to $5 \times$. Several other experiments using multi-picosecond pulselengths, large laser energies, and large focal spots have also observed a significant increase in maximum proton energies for quasi-relativistic intensities at these laser conditions. ${ }^{24-27}$

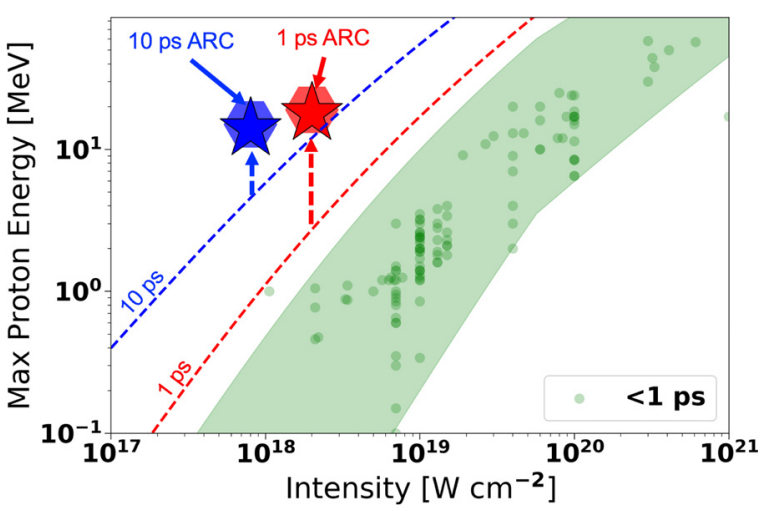

FIG. 5. Proton maximum energy vs intensity for sub-picosecond, small focal spot data from many experiments (green dots); ${ }^{22,28-38}$ the present ARC experiments at 10 and 1 ps (stars). The shaded region represents the Fuchs scaling for a region covering $0.03-0.7 \mathrm{ps}$ and a focal spot diameter of $10 \mu \mathrm{m}$. The dashed lines show the Fuchs scaling for a range of intensities at a fixed focal spot diameter of $50 \mu \mathrm{m}$ for 10 (blue dashed) and 1 ps (red dashed). The hexagonal points behind the stars are the maximum proton energies predicted by LSP simulations. 


\section{MODELING}

A three staged modeling approach as a predictive capability for particle acceleration on ARC is seen schematically in Fig. 6. This approach first utilizes the rad hydro code, HYDRA, ${ }^{39}$ to model the prepulse and the resulting preplasma developed over several nanoseconds. The particle in cell code PSC ${ }^{40}$ is then used to model the energy spectrum of electrons accelerated during the interaction of the main pulse with this preplasma, and finally, this electron source is injected into $\mathrm{LSP}^{41}$ to predict the resulting ion spectrum. This approach allows for the modeling of the very large time and spatial scales and the resulting maximum proton energies. The ability to predict the maximum proton energies makes this process an extremely valuable tool and thus allows for the examination of the physics that is responsible for exceeding conventional proton scalings for these laser intensities, energies, and focal spot conditions. A key finding from the simulation results can be seen in Fig. 6. First, it is important to realize that 1D simulations are in fact a reasonable approximation for these experiments since the focal spots are much larger than typical short-pulse laser experiments and the model is valid in the region near the center of the laser focal spot. Thus, the low density $\left(\sim 1 \% \mathrm{n}_{c}\right)$ preplasma expansion is realistic for at least $\sim 30 \mu \mathrm{m}$. The laser interaction with this long scale-length low-density preplasma allows for exploitation of super-ponderomotive electron acceleration mechanisms outlined earlier. In the figure, the electron temperature is seen to continue to rise as the multi-picosecond laser pulse drives plasma expansion. As in the experiments, the modeling predicts a hot electron tail that is $\sim 10 \times$ that expected from the ponderomotive temperature scaling. In fact, the majority of electrons accelerated in the forward direction are at the expected ponderomotive temperature. While significantly fewer $1+\mathrm{MeV}$ electrons are accelerated, they can still contain a significant fraction of the total electron population energy. Thus, it is these electrons which are ultimately responsible for driving the sheath field that accelerates protons to $10+\mathrm{MeV}$ energies.

Target normal sheath acceleration relies on the production of high energy electrons which must quickly pass through the target to set up the strong charge separation at the rear surface for proton acceleration to high (10s of $\mathrm{MeV}$ ) energies. For these intensities, the ponderomotive temperature is similar to $0.1 \mathrm{MeV}$, which is plotted for reference in Fig. 4(a). While the sheath field persists for many picoseconds, it also reaches $6.5 \mathrm{MeV}$ at 13 ps into the LSP simulations, as seen in Fig. 7(b). At this point, the ponderomotive electrons are not energetic to pass through this potential barrier in order to participate in maintaining the TNSA field. However, by this time, the superponderomotive electron tail has been well established and electrons with sufficient energy to pass through this barrier are being produced. Figure 7 (b) shows that the TNSA field can persist at $\sim 0.08 \mathrm{TV} / \mathrm{m}$ for long times, out to at least 11 ps. Since electrons below the TNSA potential cannot escape and those that do have given up a substantial portion of their energy, the measured electron spectrum seen in Fig. 4(a) is only a portion of that generated during the entirety of the laser pulse. This effect is captured in the LSP simulations as seen in Figs. 7(c) and 7(d), where the electron spectra are measured at various distances from the target as seen in Fig. 7(c) and plotted versus the input electron spectrum in (d). While the similarity of the electron temperatures suggest an expectation of an indistinguishable maximum proton energy between the 1.5 and 10 ps pulses, the timescale over which the electron temperature rises to the maximum has recently been shown to enhance the maximum proton energy. ${ }^{42}$ Since the hot electron temperature rises to a maximum on a significantly shorter time-scale for the 1.5 ps pulse, higher energy electrons can assist with the development of a stronger sheath field earlier in time and thus result in a higher maximum proton energy compared to a longer pulse-length with a similar maximum electron temperature.

\section{CONCLUSIONS}

In summary, two robust proton sources have been demonstrated on NIF's ARC laser. The maximum proton energies observed, i.e., 18 and $14 \mathrm{MeV}$ for the 1 and 10 ps pulse-lengths, respectively, far exceed the predicted proton scaling. A good conversion efficiency into protons is observed, resulting in $\sim 50 \mathrm{~J}$ into protons with energies $>3 \mathrm{MeV}$ which is an ideal source for a proton isochoric heating platform. Depositing this large amount of energy could heat a solid density sample to 100 s of eV temperatures before significant hydrodynamic expansion can occur, producing an exotic Warm Dense Matter state that could then be used for equation of state (EOS) or opacity measurements. Exceeding $14 \mathrm{MeV}$ with the shorter pulse-length enables proton probing of experiments beyond the two energies typically used

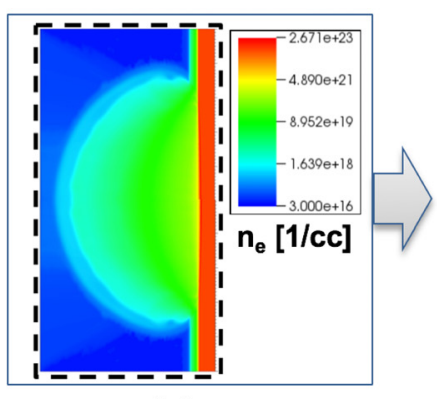

(a)

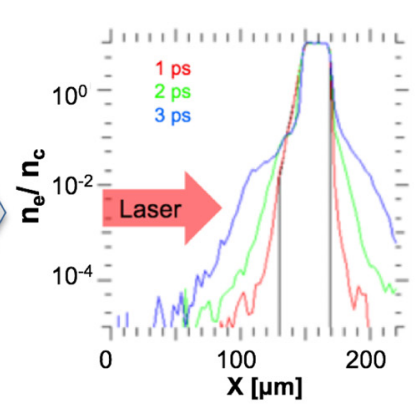

(b)

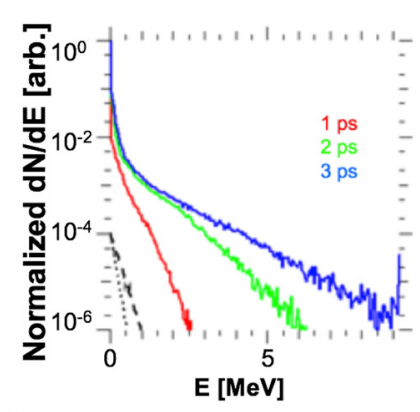

)

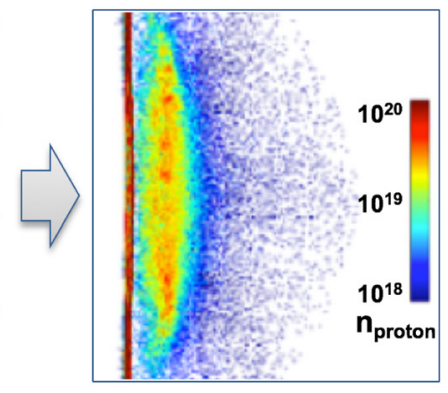

(c)

FIG. 6. A schematic of the three-stage approach for simulating proton acceleration on ARC experiments. (a) A radiation-hydrodynamics code is used to simulate the expected preplasma generated by amplified stimulated emission arriving on the target (from the left) several nanoseconds before the main pulse and the plasma expansion on the laserfacing side of the target. (b) 1D PSC particle-in-cell simulations show further expansion of a 10s of microns scale-length preplasma (left) and the development of a super-hot electron tail (right). (c) The time-dependent electron source is injected into 2D LSP simulations (from left to right), and the accelerated proton density is seen moving to the right. 
(a)

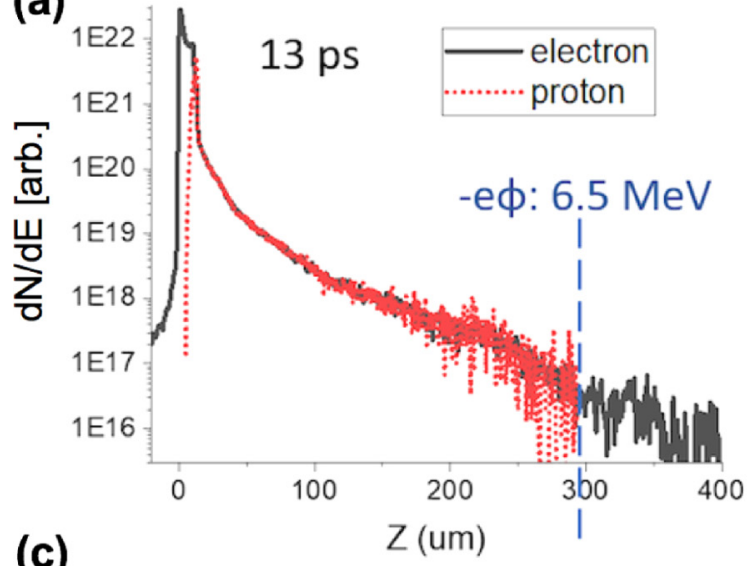

(c)

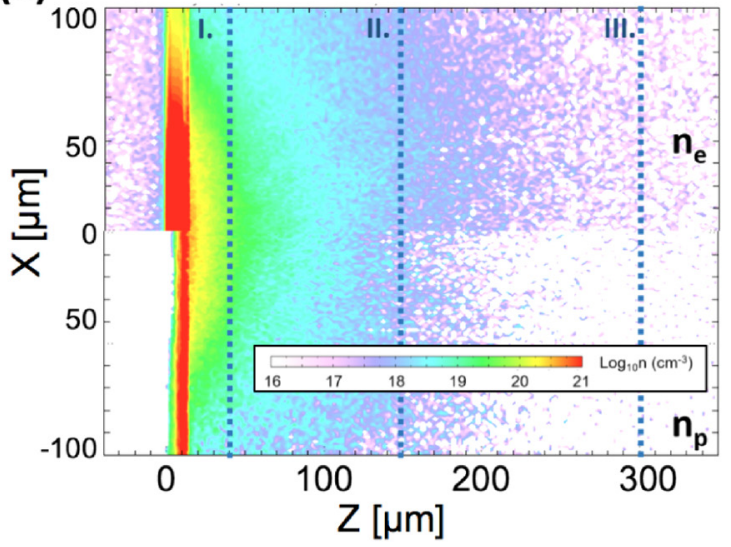

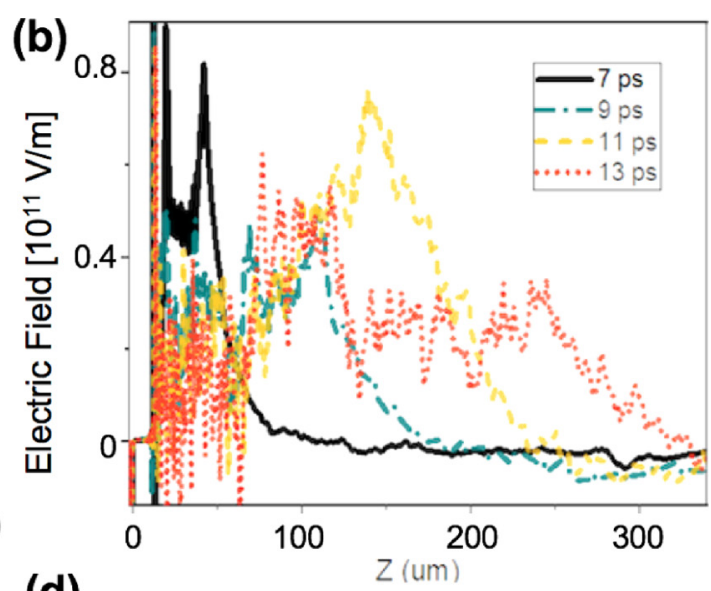

(d)

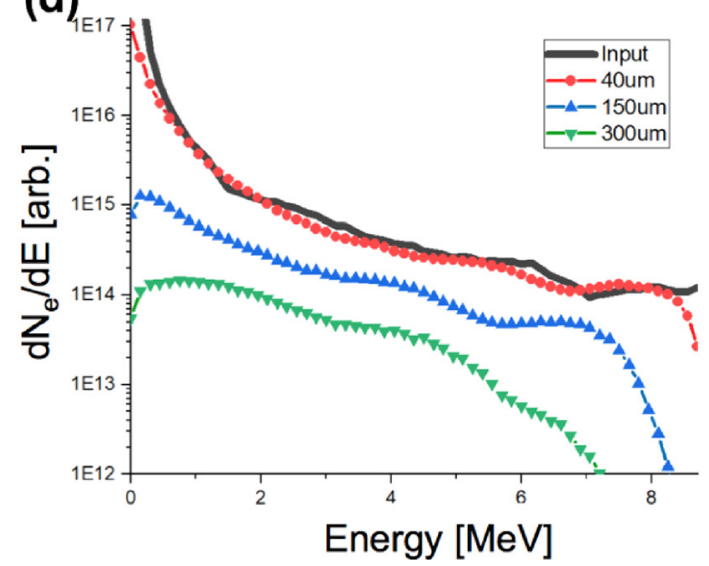

FIG. 7. LSP modeling of TNSA proton acceleration with $10 \mathrm{ps}$ at a peak intensity of $8 \times 10^{17} \mathrm{~W} / \mathrm{cm}^{2}$. (a) The proton and electron density at 13 ps, showing the position of the ion front. (b) The evolving TNSA electric field strength at various times throughout the simulation showing the persistence of a $-0.08 \mathrm{TV} / \mathrm{m}$ electric field at $11 \mathrm{ps}$ into the simulation. (c) The 2D electron and proton densities showing the position of extraction planes for extracting the escaping electron spectra shown in (d).

via exploding pushers $(14.7 \text { and } 3 \mathrm{MeV})^{43}$ and importantly uses only 4 of NIF's 192 laser beams compared to the 32-16 required to drive an implosion. The scaling of electron acceleration in this regime will be an ongoing effort and will enable a revision of the scaling models to accurately predict maximum proton energies in this new class of energetic short-pulse lasers. Several avenues have been proposed for enhancing ARC-driven proton sources. These include deliberately making a longer and higher density preplasma at the front target surface or the use of plasma optics to increase the on-target laser intensity. Future work will include the demonstration of the aforementioned proton beam applications on the NIF as well as ongoing work to understand the physics of particle acceleration and scaling with large energy, large focal spot, quasi-relativistic intensity laser conditions.

\section{ACKNOWLEDGMENTS}

The authors would like to thank the NIF ARC operations team, NIF Discovery Science management, and technical support staff for expertise and support in the execution of these experiments. This work was funded under the auspices of the U.S. Department of Energy by Lawrence Livermore National Laboratory under Contract No. DE-AC5207NA27344 with the funding support from the Laboratory Directed
Research and Development Program under tracking code 17-ERD-039 and the DOE Office of Science Early Career Research Program under SCW1651. This document was prepared as an account of work sponsored by an agency of the United States government. Neither the United States government nor Lawrence Livermore National Security, LLC, nor any of their employees makes any warranty, expressed or implied, or assumes any legal liability or responsibility for the accuracy, completeness, or usefulness of any information, apparatus, product, or process disclosed, or represents that its use would not infringe privately owned rights. Reference herein to any specific commercial product, process, or service by trade name, trademark, manufacturer, or otherwise does not necessarily constitute or imply its endorsement, recommendation, or favoring by the United States government or Lawrence Livermore National Security, LLC. The views and opinions of authors expressed herein do not necessarily state or reflect those of the United States government or Lawrence Livermore National Security, LLC, and shall not be used for advertising or product endorsement purposes.

\section{REFERENCES}

${ }^{1}$ L. Waxer, D. Maywar, J. Kelly, T. Kessler, B. Kruschwitz, S. Loucks, R. McCrory, D. Meyerhofer, S. Morse, C. Stoeckl et al., Opt. Photonics News 16, 30 (2005). 
${ }^{2}$ J. Kawanaka, N. Miyanaga, H. Azechi, T. Kanabe, T. Jitsuno, K. Kondo, Y. Fujimoto, N. Morio, S. Matsuo, Y. Kawakami et al., J. Phys.: Conf. Ser. 112, 032006 (2008).

${ }^{3}$ N. Blanchot, G. Behar, T. Berthier, B. Busserole, C. Chappuis, C. DamiensDupont, P. Garcia, F. Granet, C. Grosset-Grange, J.-P. Goossens et al., EPJ Web Conf. 59, 07001 (2013).

${ }^{4}$ H. Chen, M. Hermann, D. Kalantar, D. Martinez, P. Di Nicola, R. Tommasini, O. Landen, D. Alessi, M. Bowers, D. Browning et al., Phys. Plasmas 24, 033112 (2017).

${ }^{5}$ A. J. Mackinnon, P. K. Patel, M. Borghesi, R. C. Clarke, R. R. Freeman, H. Habara, S. P. Hatchett, D. Hey, D. G. Hicks, S. Kar, M. H. Key, J. A. King, K. Lancaster, D. Neely, A. Nikkro, P. A. Norreys, M. M. Notley, T. W. Phillips, L. Romagnani, R. A. Snavely, R. B. Stephens, and R. P. J. Town, Phys. Rev. Lett. 97, 045001 (2006).

${ }^{6}$ P. Patel, A. Mackinnon, M. Key, T. Cowan, M. Foord, M. Allen, D. Price, H. Ruhl, P. Springer, and R. Stephens, Phys. Rev. Lett. 91, 125004 (2003).

${ }^{7}$ J. Kim, B. Qiao, C. McGuffey, M. Wei, P. Grabowski, and F. Beg, Phys. Rev. Lett. 115, 054801 (2015).

${ }^{8}$ A. Alejo, A. Krygier, H. Ahmed, J. Morrison, R. Clarke, J. Fuchs, A. Green, J. Green, D. Jung, A. Kleinschmidt et al., Plasma Phys. Controlled Fusion 59, 064004 (2017).

${ }^{9}$ D. Higginson, J. McNaney, D. Swift, T. Bartal, D. Hey, R. Kodama, S. Le Pape, A. Mackinnon, D. Mariscal, H. Nakamura, N. Nakanii, K. A. Tanaka, and F. N. Beg, Phys. Plasmas (1994-present) 17, 100701 (2010).

${ }^{10}$ S. Wilks, A. Langdon, T. Cowan, M. Roth, M. Singh, S. Hatchett, M. Key, D. Pennington, A. MacKinnon, and R. Snavely, Phys. Plasmas (1994-present) 8, 542 (2001).

${ }^{11}$ S. C. Wilks and W. L. Kruer, IEEE J. Quantum Electron. 33, 1954 (1997).

${ }^{12}$ E. Esarey, C. Schroeder, and W. Leemans, Rev. Mod. Phys. 81, 1229 (2009).

${ }^{13}$ A. Pukhov, Z.-M. Sheng, and J. Meyer-ter Vehn, Phys. Plasmas 6, 2847 (1999).

${ }^{14}$ A. Arefiev, V. Khudik, A. Robinson, G. Shvets, L. Willingale, and M. Schollmeier, Phys. Plasmas 23, 056704 (2016).

${ }^{15}$ B. Paradkar, S. Krasheninnikov, and F. Beg, Phys. Plasmas 19, 060703 (2012).

${ }^{16}$ A. Sorokovikova, A. Arefiev, C. McGuffey, B. Qiao, A. Robinson, M. Wei, H. McLean, and F. Beg, Phys. Rev. Lett. 116, 155001 (2016).

${ }^{17}$ H. Chen, A. J. Link, R. van Maren, P. K. Patel, R. Shepherd, S. C. Wilks, and P. Beiersdorfer, Rev. Sci. Instrum. 79, 10E533 (2008).

${ }^{18}$ D. Mariscal, G. Williams, H. Chen, S. Ayers, N. Lemos, S. Kerr, and T. Ma, "Calibration of proton dispersion for the NIF electron positron proton spectrometer (NEPPS) for short-pulse laser experiments on the NIF ARC” Rev. Sci. Instrum. 89, $10 \mathrm{I} 145$ (2018).

${ }^{19}$ D. Casey, J. Frenje, M. Gatu Johnson, F. Séguin, C. Li, R. Petrasso, V. Y. Glebov, J. Katz, J. Magoon, D. Meyerhofer et al., Rev. Sci. Instrum. 84, 056311 (2013).

${ }^{20}$ F. Séguin, N. Sinenian, M. Rosenberg, A. Zylstra, M.-E. Manuel, H. Sio, C. Waugh, H. Rinderknecht, M. G. Johnson, J. Frenje et al., Rev. Sci. Instrum. 83, 10D908 (2012).

${ }^{21}$ D. Hey, M. Key, A. Mackinnon, A. MacPhee, P. Patel, R. Freeman, L. Van Woerkom, and C. Castaneda, Rev. Sci. Instrum. 79, 053501 (2008).
${ }^{22}$ J. Fuchs, P. Antici, E. d'Humieres, E. Lefebvre, M. Borghesi, E. Brambrink, C. Cecchetti, M. Kaluza, V. Malka, M. Manclossi, S. Meyroneinc, P. Mora, J. Schreiber, T. Toncian, H. Pépin, and P. Audebert, Nat. Phys. 2, 48 (2006).

${ }^{23}$ P. Mora, Phys. Rev. Lett. 90, 185002 (2003).

${ }^{24}$ K. Flippo, T. Bartal, F. Beg, S. Chawla, J. Cobble, S. Gaillard, D. Hey, A. MacKinnon, A. MacPhee, P. Nilson et al., J. Phys.: Conf. Ser. 244, 022033 (2010).

${ }^{25}$ A. Yogo, K. Mima, N. Iwata, S. Tosaki, A. Morace, Y. Arikawa, S. Fujioka, T. Johzaki, Y. Sentoku, H. Nishimura et al., Sci. Rep. 7, 42451 (2017).

${ }^{26}$ L. Gao, P. M. Nilson, W. Theobald, C. Stoeck, 1. C. Dorrer, T. C. Sangster, D. D. Meyerhofer, L. Willingale, and K. M. Krushelnick, "Measurements of proton generation with intense kilojoule laser pulses on omega," in Proc. of 52nd Annual Meeting of the APS Division of Plasma Physics (American Phys. Soc., 2010). Available at http://meetings.aps.org/link/BAPS.2010.DPP.XO6.1.

${ }^{27}$ N. Iwata, K. Mima, Y. Sentoku, A. Yogo, H. Nagatomo, H. Nishimura, and H. Azechi, Phys. Plasmas 24, 073111 (2017).

${ }^{28}$ R. A. Snavely, M. H. Key, S. P. Hatchett, T. E. Cowan, M. Roth, T. W. Phillips, M. A. Stoyer, E. A. Henry, T. C. Sangster, M. S. Singh, S. C. Wilks, A. MacKinnon, A. Offenberger, D. M. Pennington, K. Yasuike, A. B. Langdon, B. F. Lasinski, J. Johnson, M. D. Perry, and E. M. Campbell, Phys. Rev. Lett. 85, 2945 (2000).

${ }^{29}$ K. Zeil, S. Kraft, S. Bock, M. Bussmann, T. Cowan, T. Kluge, J. Metzkes, T. Richter, R. Sauerbrey, and U. Schramm, New J. Phys. 12, 045015 (2010).

${ }^{30}$ M. Borghesi, D. Campbell, A. Schiavi, O. Willi, A. Mackinnon, D. Hicks, P. Patel, L. Gizzi, M. Galimberti, and R. Clarke, Laser Part. Beams 20, 269 (2002).

${ }^{31}$ S. Fritzler, V. Malka, G. Grillon, J.-P. Rousseau, F. Burgy, E. Lefebvre, E. d'Humieres, P. McKenna, and K. Ledingham, Appl. Phys. Lett. 83, 3039 (2003).

${ }^{32}$ T. Fujii, Y. Oishi, T. Nayuki, Y. Takizawa, K. Nemoto, T. Kayoiji, K. Horioka, Y. Okano, Y. Hironaka, K. G. Nakamura et al., Appl. Phys. Lett. 83, 1524 (2003).

${ }^{33}$ Y. Oishi, T. Nayuki, T. Fujii, Y. Takizawa, X. Wang, T. Yamazaki, K. Nemoto, T. Kayoiji, T. Sekiya, K. Horioka et al., Phys. Plasmas 12, 073102 (2005).

${ }^{34}$ I. Spencer, K. Ledingham, P. McKenna, T. McCanny, R. Singhal, P. Foster, D. Neely, A. Langley, E. Divall, C. Hooker et al., Phys. Rev. E 67, 046402 (2003).

${ }^{35}$ A. Mackinnon, Y. Sentoku, P. Patel, D. Price, S. Hatchett, M. Key, C. Andersen, R. Snavely, and R. Freeman, Phys. Rev. Lett. 88, 215006 (2002).

${ }^{36}$ L. Robson, P. Simpson, R. Clarke, K. Ledingham, F. Lindau, O. Lundh, T. McCanny, P. Mora, D. Neely, C.-G. Wahlström, M. Zepf, and P. McKenna, Nat. Phys. 3, 58 (2007).

${ }^{37}$ M. Kaluza, J. Schreiber, M. Santala, G. Tsakiris, K. Eidmann, J. Meyer-ter Vehn, and K. Witte, Phys. Rev. Lett. 93, 045003 (2004).

${ }^{38}$ G. Malka and J. Miquel, Phys. Rev. Lett. 77, 75 (1996).

${ }^{39}$ M. Marinak, G. Kerbel, N. Gentile, O. Jones, D. Munro, S. Pollaine, T. Dittrich, and S. Haan, Phys. Plasmas 8, 2275 (2001).

${ }^{40} \mathrm{M}$. Bonitz and D. Semkat, Introduction to Computational Methods in Many Body Physics (Rinton Press, Inc., 2006).

${ }^{41}$ D. R. Welch, D. Rose, B. Oliver, and R. Clark, Nucl. Instrum. Methods Phys. Res. Sect. A 464, 134 (2001).

${ }^{42}$ J. Kim, A. Kemp, S. Wilks, D. Kalantar, S. Kerr, D. Mariscal, F. Beg, C. McGuffey, and T. Ma, Phys. Plasmas 25, 083109 (2018).

${ }^{43}$ C. Li, F. Séguin, J. Frenje, M. Rosenberg, R. Petrasso, P. Amendt, J. Koch, O. Landen, H. Park, H. Robey et al., Science 327, 1231 (2010). 